\title{
X-RAY EMISSION FROM SOLAR NEIGHBOURHOOD FLARE STARS
}

\author{
R. PALLAVICINI ${ }^{1}$, L. STELLA ${ }^{2}$ and G. TAGLIAFERRI ${ }^{3}$ \\ 1) Osservatorio Astrofisico di Arcetri, Firenze, Italy \\ 2) Dipartimento di Fisica, Universitd di Roma, Italy \\ 3) EXOSAT Observatory, ESTEC, Noordwijk, The Netherlands
}

\begin{abstract}
A brief summary is given of a comprehensive analysis of EXOSAT observations of solar neighbourhood flare stars. Special attention is devoted to the discussion of quiescent X-ray emission and time variability.
\end{abstract}

\section{Introduction}

During its operational lifetime (May 1983-April 1986) the European satellite EXOSAT obtained many valuable observations of UV Ceti-type flare stars at X-ray wavelengths. The EXOSAT sample comprises 23 different objects from the flare star catalogue of Pettersen (1976). A number of them were observed on several occasions. If we also include a few other flare stars detected serendipitously, the sample comprises more than $\mathbf{4 0}$ separate observations for a total monitoring time of nearly 300 hours. The most significant aspect of these data is that about one half of them were long continuous observations extending over periods of many hours, without the data gaps that were usually associated to previous low-orbit satellites. This allowed us to study quiescent and flaring X-ray emission from UV Ceti-type flare stars in a much better way than it had been possible before (see Haisch 1983, Agrawal et al. 1986, Ambruster et al. 1987 for previous Einstein observations)

We have carried out a comprehensive analysis of EXOSAT observations of flare stars, using data from the Low Energy (LE: $0.05-2 \mathrm{KeV}$ ) and Medium Energy (ME: 1-10 KeV) experiments on EXOSAT. A full account of this study is in press in Astronomy and Astrophysics; here we give only a short summary of our work and present the most significant results.

\section{Quiescent emission}

The quiescent $X$-ray luminosities for the stars in our sample span a range of nearly a factor 500 (from $1.4 \times 10^{27} \mathrm{erg} \mathrm{s}^{-1}$ for Prox Cen to $6.7 \times 10^{29} \mathrm{erg} \mathrm{s}^{-1}$ for YY Gem), in spite of the fact that these stars do not differ much in spectral type. We have not found any obvious correlation between the quiescent $\mathrm{X}$-ray luminosity and the rotation rate of the star, in contrast with what could be expected from simple dynamo models. Instead, we have found a very good correlation between X-ray quiescent luminosity and bolometric luminosity. This is shown in Fig. 1. The correlation coefficient is 0.95 for 24 data points and the best fit relationship is

$$
\log \mathrm{L}_{\mathrm{x}}=-9.83+1.21 \log \mathrm{L}_{\text {bol }}
$$

This dependence of coronal activity on bolometric luminosity suggests a dependence on the stellar radius as would be expected if activity in these stars occurs in a "saturation" regime.

In contrast to the large variations observed between different objects, the quiescent $\mathrm{X}$-ray emission of any given star appears to change little from one observation to the other. For stars observed repeatedly by EXOSAT, the observed variations were always less than a factor $\approx 2$, and more typically did not exceed amplitudes of $\approx 20-30 \%$. The time variations observed in the course of any individual observation were 
also typically small (less than $\approx 50 \%$ ), except during flares. The latter show a wide range of amplitudes with respect to the quiescent level, from less than a factor 2 to more than one order of magnitude.

Twenty stars in our sample were observed previously with Einstein. We find a very good correlation between the EXOSAT and Einstein average fluxes, the difference being less than a factor $\approx 2$ for most sources. This is comparable to the typical error on the determination of EXOSAT to Einstein flux ratios. For only 3 stars we found somewhat larger differences (a factor 3 to 4). This suggests that long-term variations of coronal X-ray emission (as might be expected from activity cycles) are probably small for these stars, at least in the spectral bands accessible to EXOSAT and Einstein.

\section{Short-term variability}

We have carried out a detailed time analysis of EXOSAT LE observations in search of low-amplitude shortterm variability as might be expected from "microflaring" activity. Time variability has been investigated by us by applying several different techniques, including variance, correlation and power spectra analysis. For most stars in our sample we find substantial variability over a variety of different times scales (from a few minutes to hours). The observed variability appears in the form of either individual (usually sporadic) flares or of more gradual variations (on times scales of tens of minutes to hours). This result is consistent with what found previously from Einstein observations (Ambruster et al. 1987).

By contrast, we do not find evidence in the EXOSAT data for continuous low amplitude variability on time scales shorter than a few hundred seconds. More specifically, we do not confirm the low-level "microflaring" variability, consisting of a succession of events lasting from tens of seconds to several minutes and with characteristic energies of $\approx 2 \times 10^{30} \mathrm{erg}$, that was reported by Butler et al. (1986).

In the case of UV Ceti, the availability of four different EXOSAT observations allows a more detailed investigation of its variability properties. To this end, we have combined the four observations (after excluding a strong flare that was present in one of them) and we have computed an average power spectrum for the entire data set. This is shown in Fig. 2, where the power spectrum has been normalized in such a way that the counting statistics noise corresponds to a power of 2 . As shown in the figure, significant power above the noise level is detected only between frequencies of $\approx 3 \times 10^{-5}$ and $\approx 2 \times 10^{-3} \mathrm{~Hz}$. No evidence for excess power at higher frequencies is found. This implies that the shorter variability time scales of UV Ceti revealed by the EXOSAT observations are on the order of a few hundred seconds. This is in agreement with what found independently by Collura et al. (1988) using a different variability analysis.

\section{Flaring activity}

A few dozens flares have been observed by EXOSAT from UV Ceti-type stars. For a few of them, ME data were obtained in addition to the LE ones. This allowed spectral analysis of the flare and determination of relevant physical parameters such as coronal temperatures and emission measures.

The observed flares cover a broad range of total X-ray energies (from $\approx 3 \times 10^{30} \mathrm{erg}$ to $\approx 1 \times 10^{34} \mathrm{erg}$ ) and have a variety of different time scales (from a few minutes to hours). There is evidence in the EXOSAT data for at least two different types of stellar flares, i. e.: a) impulsive flares, which are reminiscent of compact flares on the Sun; and b) long-decay flares, which are reminiscent of long-duration solar tworibbon flares.

Temperatures derived from spectral analysis of stellar flares are in the range from $\approx 2 \times 10^{7} \mathrm{~K}$ to $\approx 4 \mathrm{x}$ $10^{7} \mathrm{~K}$, similar to the typical temperatures found for solar X-ray flares. The emission measures are in the range from $\approx 1 \times 10^{51} \mathrm{~cm}^{-3}$ to $\approx 1 \times 10^{53} \mathrm{~cm}^{-3}$, much larger than for solar flares. There is evidence that the plasma is first heating and then cooling during the evolution of the flare. Moreover, the high energy flux appears to precede the low energy flux by a few to several minutes, as also typically observed in solar flares. Within our limited sample there is no obvious correlation of flare temperatures and time scales with other stellar parameters. We found instead a correlation between quiescent X-ray emission and flare energy, the largest flares occurring only on the most active stars.

The average rate of detection of X-ray flares was typically $\approx 1$ flare every $\approx 10$ hours for the stars observed by EXOSAT. However, flare detectability strongly depends on the quiescent X-ray level of the star and decreases sharply as the latter increases. The available data do not allow the determination of the 
flare frequency distribution vs. energy for each individual star in our sample. However, by assuming that all stars in the sample obey the same relationship, we found that the flare frequency distribution can be approximated at high flare energies as a power law (see Fig. 3), where $N(>E)$ is the number of flares with total X-ray energy larger than $E$ and $\alpha$ is the power-law index which turns out to be $0.7 \pm 0.1$. At lower flare energies the distribution flattens owing to detection threshold effects. The shape of the derived flare frequency distribution is consistent with that found for optical flares (for which $\alpha$ varies from 0.4 to 1.2 depending on the star).

\section{References}

Agrawal, P.C., Rao, A.R., and Sreekantan, B.V.: 1986, Montly Not. Roy. Astron. Soc. 219, 225

Ambruster, C.W., Sciortino, S., and Golub, L.: 1987, Astrophys. J. Suppl. 65, 273

Butler, C.J., Rodond, M., Foing, B.H., and Haisch, B.M.: 1986, Nature 321, 679.

Collura, A., Pasquini, L., and Schmitt, J.H.M.M.: 1988, Astron. Astrophys. 205, 197

Haisch, B.M.: 1983, in Activity in Red-dwarf Stars, eds. P.B. Byrne and M. Rodonò, Dordrecht: Reidel Publ. Co., p. 255

Pettersen, B.R.: 1976, Catalogue of Flare Star Data, Inst. Theor. Astrophys., Blindern, Report No. 46

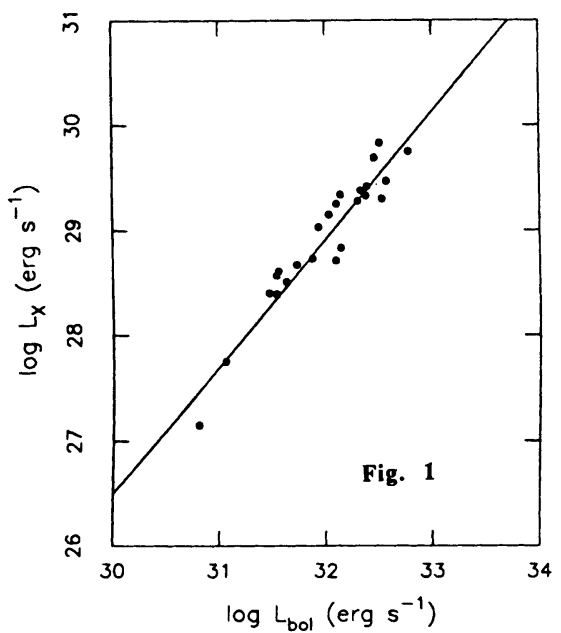

UV Ceti

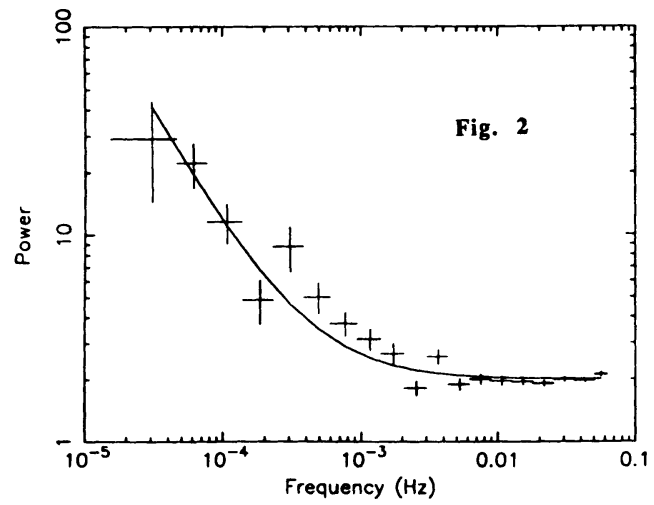

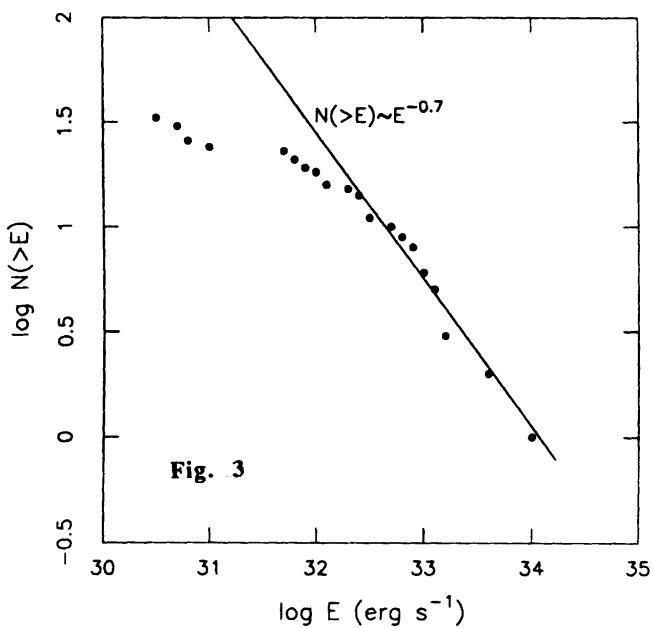

Fig. 1: X-ray quiescent luminosity versus bolometric luminosity

Fig. 2: Average power spectrum of UV Ceti

Fig. 3: X-ray flare frequency distribution 
GIAMPAPA: Since $\mathrm{I}_{X}$ is correlated with $\mathrm{I}_{\mathrm{bo}}$ but not with rotation period does this really mean that $I_{X}$ is correlated with radius (surface area), i.e.the surface is "saturated" with magnetic flux tubes at high rotation rates?

PALLAVICINI: A correlation of $\mathrm{L}_{X}$ with radius for very active flare stars is probably the most plausible interpretation at present. If this occurs, certainly we must be in a "saturated" stage, since no such correlation between $L_{\mathrm{X}}$ and $\mathrm{L}_{\mathrm{b} .01}$ is observed for non-flaring dM stars.

GIAMPAPA: DO you see periodic structure in the quiescent or flare $\mathrm{x}$-ray emission?

PALLAVICINI: A detailed variability study carried out over our EXOSAT observations did not show any periodic structure in the quiescent or flare emission.

HERBST: Concerning the $I_{X}-I_{b o 1}$ correlation, we find an $L_{H \text { Hot }}-L_{b o 1}$ relation that looks similar based on an H-alpha photometry survey of about $350 \mathrm{dM}$ stars at van vleck Observatory. It suggested to us that there was a maximum fraction of $\mathrm{L}_{\mathrm{bol}}$ which could go into $\mathrm{L}_{\mathrm{H} \alpha}$ and that fraction was more or less independent of $\mathrm{L}_{\mathrm{bol}}$. Can the same thing be said of $L_{X}$ ? Does the fraction of $I_{b o 1}$ which goes into $L_{X}$ (i.e. $I_{X} / L_{b o 1}$ ) vary with any other parameter related to stellar rotation?

PALLAVICINI: We do not find any significant correlation of $I_{\mathrm{X}} / \mathrm{L}_{\mathrm{bol}}$ with rotation period or velocity. More likely we are in a "saturation" regime as you suggested, with $\mathrm{L}_{\mathrm{X}} / \mathrm{L}_{\text {bo }} \tilde{\Gamma}^{0.001}$ for active flaring stars.

LANG: What is the large variation (two order of magnitude) of quiescent $\mathrm{X}$-ray emission due to - a variation in distance, or the temperature and emission measure of the $\mathrm{X}$-ray emitting plasma?

PALLAVICINI: It does not depend on distance. There is a correlation of $\mathrm{X}$-ray luminosity with bolometric luminosity, or probably stellar radius, and also emission measure variations should play a role.

LANG: Bigger stars probably have bigger coronal loops or more small ones, hence a greater emission measure for comparable electron densities and greater $\mathrm{X}$-ray luminosity.

GRININ: What is the typical ratio of the flare energy in soft $x$-ray and optical?

PALLAVICINI: If you mean the ratio $L_{\mathrm{bpt}} / \tau_{\mathrm{X}}$ for any given flare, I do not have an easy answer at hand. This implies simultaneous observations of flares at $X$-ray and optical wavelengths and only a few of the EXOSAT flares were observed simultaneously in the optical. My impression is that this ratio can vary quite a bit from one flare to another. I doubt that we can even talk of a "typical" $I_{X} / \tau_{\text {opt }}$ ratio. Moreover, I expect that this ratio changes considerably during the evolution of any single flare. 
BENZ: Is there a correlation between soft $X$-ray emission of $M$ dwarfs with their quiescent radio emission?

PALLAVICINI: There are only a handful of flare stars for which real "quiescent" radio emission has been detected (at levels of about $1 \mathrm{mJy}$ or less). There is no obvious correlation between $\mathrm{X}$-ray luminosity and quiescent radio luminosity for the limited sample available at present. Detailed comparison of $X$-ray and radio emission for 4 stars observed by us with EXOSAT and VLA (Kundu et al. 1988) also indicates poor correlation. More observations in the radio would be useful to address this question. 